\title{
Simulation of Analog Modulation and Demodulation Techniques in Virtual Instrumentation and Remote Lab
}

\author{
https://doi.org/10.3991/ijoe.v13i10.7575 \\ Nehru Kandasamy( $\left.{ }^{\bowtie}\right)$, Nagarjuna Telagam, V.R Seshagiri Rao \\ Institute of Aeronautical Engineering, Hyderabad, India. \\ nnehruk@gmail.com \\ T.S.Arulananth \\ MLR Institute of Technology, Hyderabad, India.
}

\begin{abstract}
An analog method to modulate and demodulate signals is presented in this short paper. This paper discusses modulation techniques with the LabVIEW-based RF communications package, developed for the illustration of different modulation types in undergraduate virtual instrumentation labs. The tool can generate and display the time and frequency domain behaviours of modulated signals with the help of spectral and tone measurements icon. All the modulation parameters can be specified by the user. This signal processing tool in the block diagram is also enables adding noise to a modulated signal, and selecting among different types of baseband pulseshaping filters. The transition between the virtual and the real world is accomplished via data-acquisition cards, installed in PCs. The results of simulation show that the interface of each system is beautiful, the parameters are easy to adjust and the process of modulation-demodulation is simpler than the traditional system which is designed by hardware circuit. This Amplitude modulation is tested in NI USRP RIO 2920 as transmitter. The carrier frequency used is $555-1650 \mathrm{KHz}$ for amplitude modulation and the carrier frequency is 88-108 MHz in India.
\end{abstract}

Keywords-NI USRP RIO 2920, amplitude and frequency modulation, Signal processing

\section{Introduction}

Communication is the process of exchanging information. The encoded symbols transmitted in the AWGN channel, at the receiver information is decoded and which recreates the original message signal [1]. Modulation technique is used to reduce the antenna height and also increases the range of communication, multiplexing and improves quality of reception [2]. The modulation schemes are broadly classified into two categories such as continuous and digital modulations [3].The topic for implementation is Amplitude \& Frequency modulation followed by demodulation. As literature survey shows that few papers are available to describe about basic voice/signal modulation and demodulation in LabVIEW and TMS320C6748 DSP kit [4]. Lab- 
VIEW is a development environment for problem solving, accelerated productivity and continual innovation. Hardware integration can be done rapidly which aids to acquire and visualize data sets from virtual $\mathrm{I} / \mathrm{O}$ devices and real time signals. It is combined with graphical programming syntax that reduces programming time [5]. LabVIEW programs are called Virtual Instruments or VIs, because their appearance and operation imitate physical instruments such as oscilloscopes, multimeters, signal generators and other basic electronic input/output devices [6]. Graphical user interface system is explained for analog data.[7].

\section{Implementation}

With the help of available tool box named signal processing in block diagram the simulation signal icon. The user can specify the parameters such as amplitude, frequency and phase. The user can also add the noise to the signal.

\subsection{Simulation of Amplitude modulation and demodulation}

$$
s(t)=A_{c}\left(1+K_{a} A_{m} \cos 2 \pi f_{m} t\right) \cos 2 \pi f_{c} t
$$

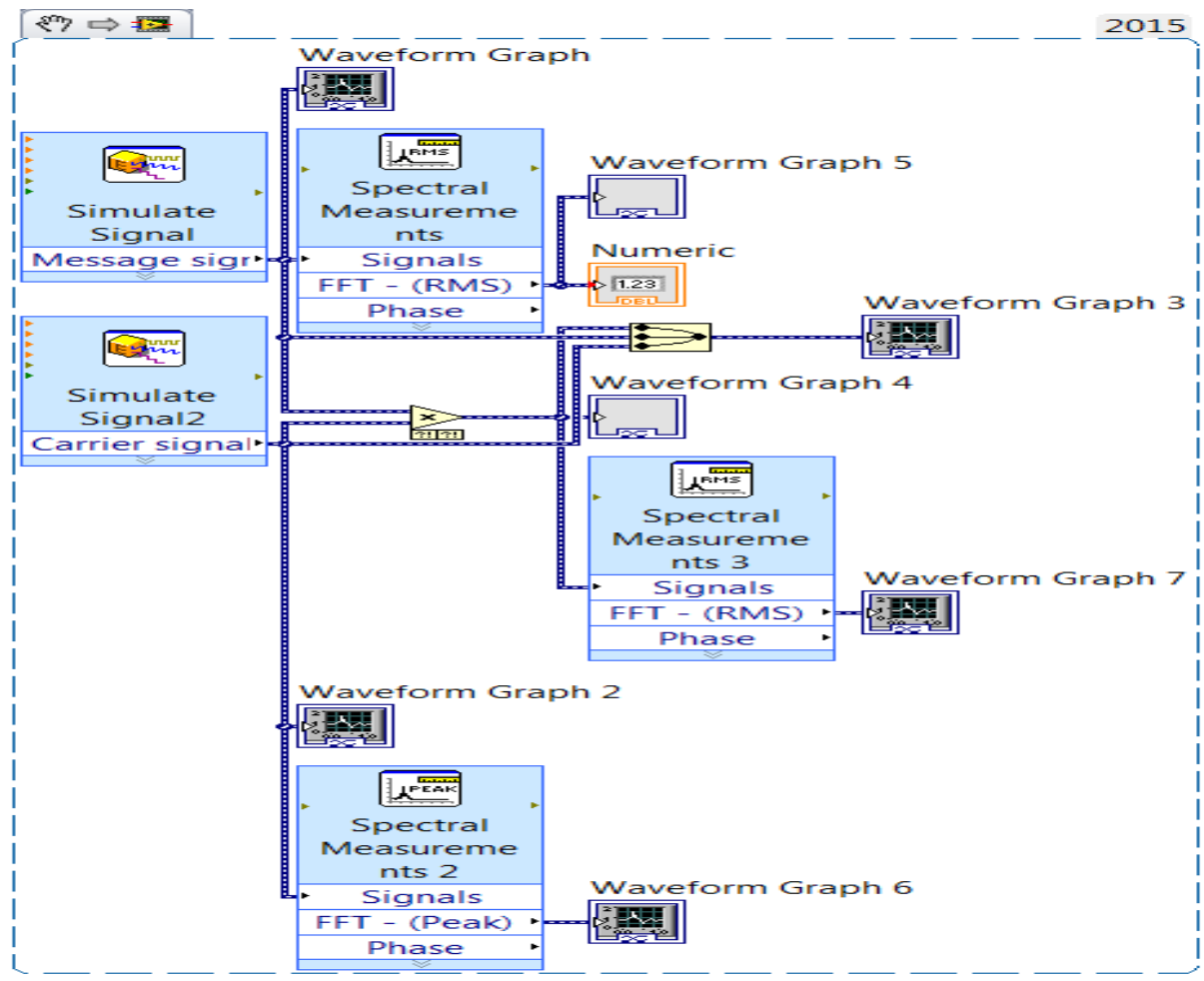

Fig. 1. VI Snippet of Amplitude modulation 
Fig 1 explains the operation of amplitude modulation in which the amplitude of carrier signal is varied in accordance with message signal amplitude variations. The spectral measurements give the frequency domain representation in linear value.

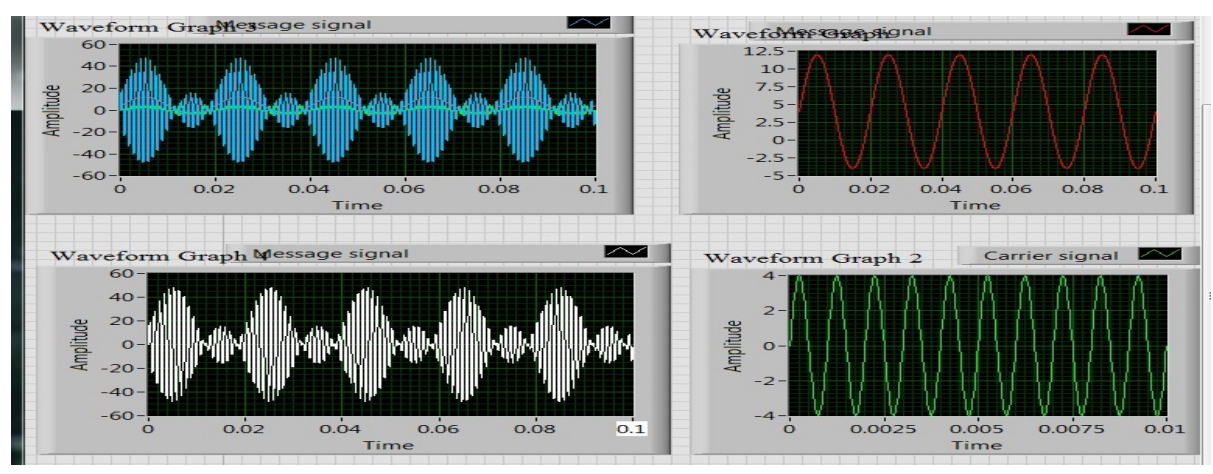

Fig. 2. Front Panel output of amplitude modulation

Fig 2 shows the ouput. With the help of graph palette the waveforms can be analyzsed in time and frequency domain clearly. The user can verify different modulation schemes such as under modulation, critical modulation and over modulation.

\subsection{Square law modulator}

The standard equation of the square law device with modulator is given as

$$
s(t)=a_{0}(m(t)+c(t))+a_{1}(m(t)+c(t))^{2}
$$

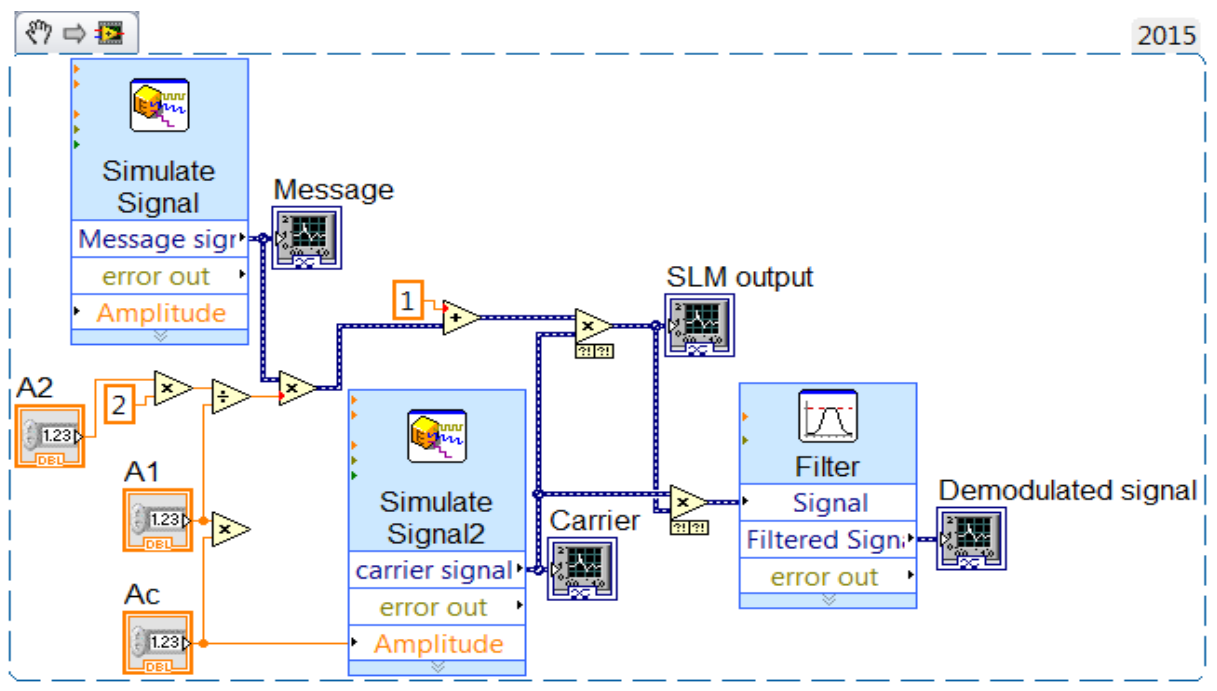

Fig. 3. VI Snippet of Square law modulator 
Fig 3 gives the implementation of amplitude modulation using square law device. The parameters $a_{0}$ and $a_{1}$ will decide the AM output because amplitude sensitivity

$$
k_{a}=2 a_{1} / a_{0}
$$

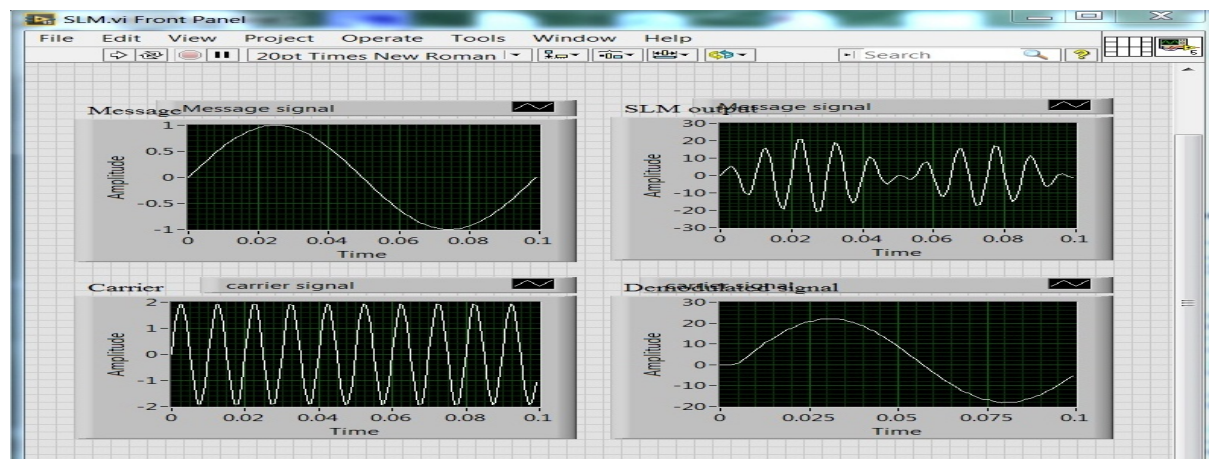

Fig. 4. Front panel output of square law modulator

Fig 4 shows the ouput of modulator and demodulation. The demodulation of the signal is done with respect to filter with butterworth type of order 5, and used as low pass filter with user specified cut off frequency.

\subsection{Double Side band Suppressed carrier}

$$
s(t)=m(t) . c(t)
$$

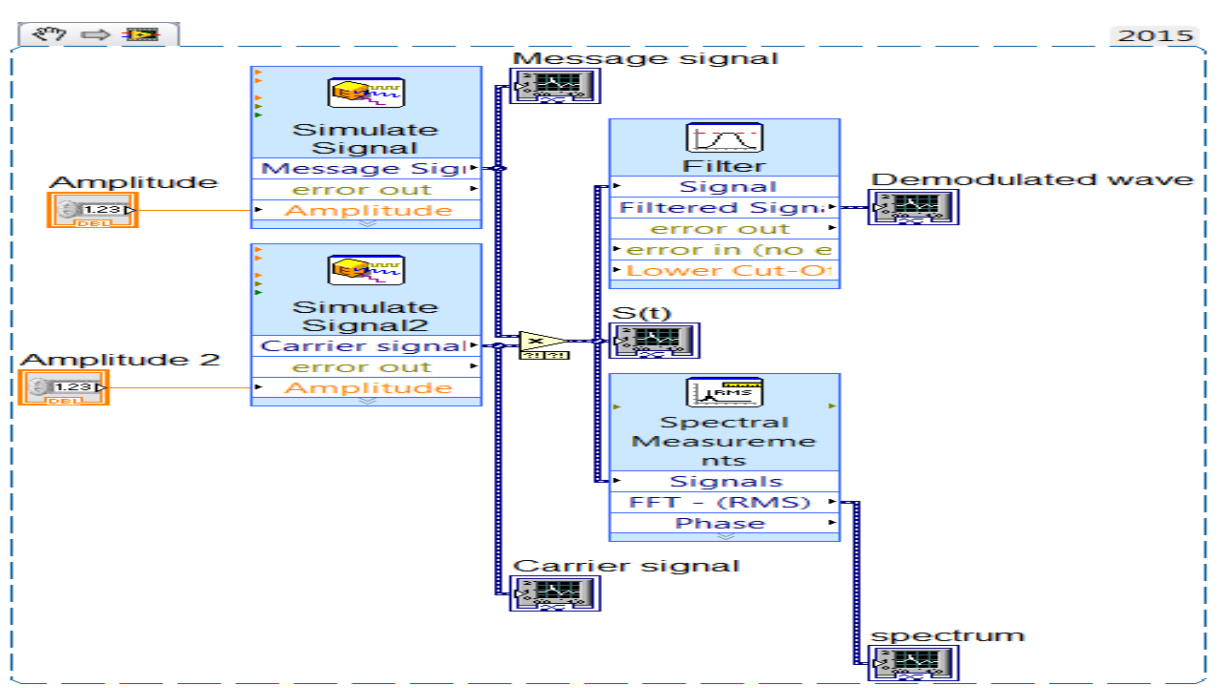

Fig. 5. VI Snippet of Double side band Suppressed carrier 
Fig 5 shows the implementation of balanced modulator. The modulation parameters are specified by the user. It is nothing but product of carrier signal with message signal and the spectral measurements icon gives the frequency domain representation. $\mathrm{m}(\mathrm{t})$ is the modulating signal and $\mathrm{c}(\mathrm{t})$ is carrier signal.

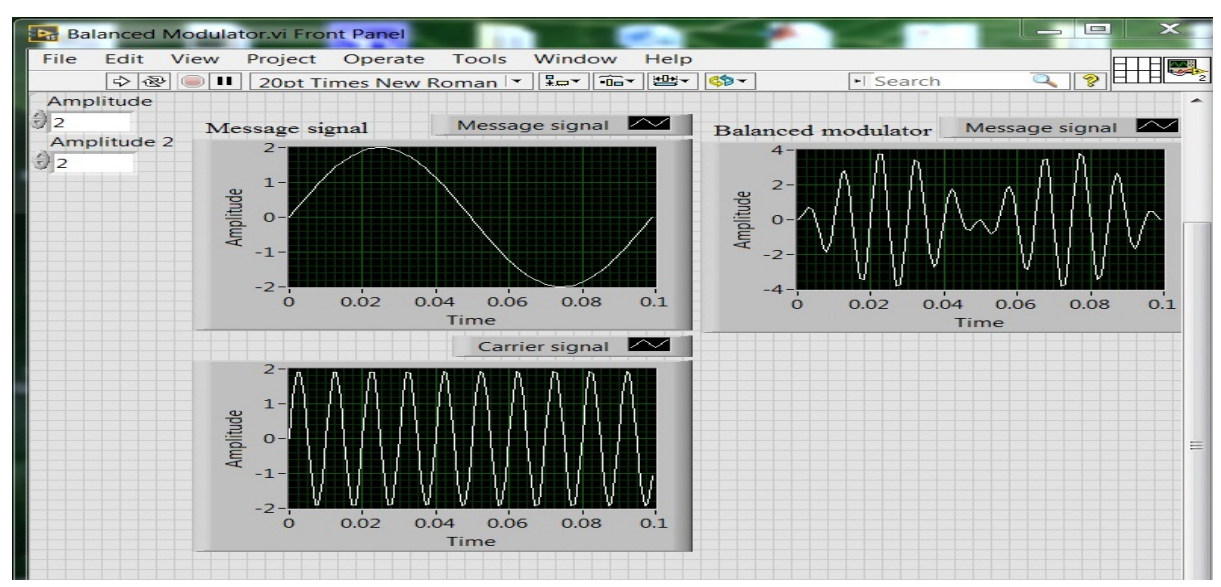

Fig. 6. Front panel output of balanced modulator

Fig 6 shows the front panel output. It shows three waveform graphs. The balanced modulator output gives the perfect modulation index i.e the ratio of amplitude of message signal with amplitude of carrier signal.

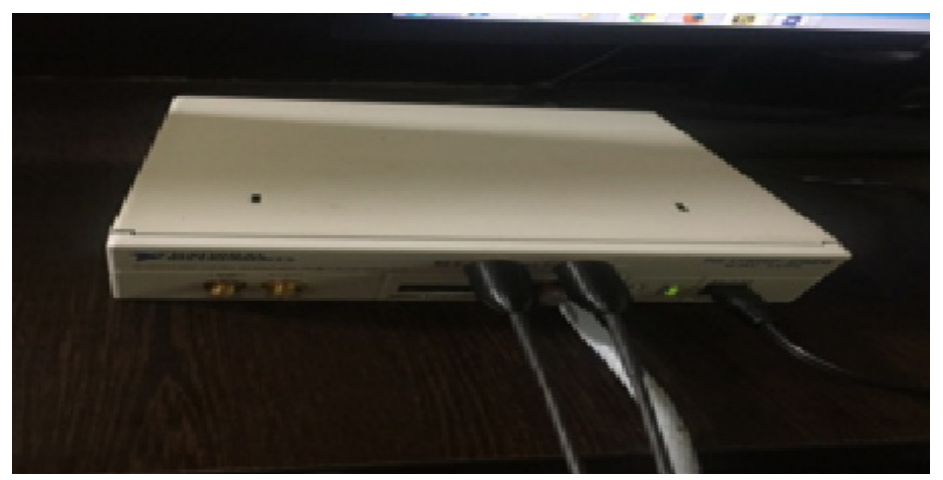

Fig. 7. NI USRP RIO 2920

Fig 7 shows the NI USRP RIO 2920 which is used in laboratory. It will operate at 23 degrees ambient temperature. The USRP-2920 is a tunable RF transceiver with a high-speed analog-to-digital converter and digital-to-analog converter for streaming baseband I and Q signals to a host PC over 1/10 Gigabit Ethernet. SRP-2920 for the following applications: white space; broadcast FM; public safety; land-mobile, lowpower unlicensed devices on industrial, scientific, and medical (ISM) bands; sensor networks; cell phone; amateur radio; or GPS. 


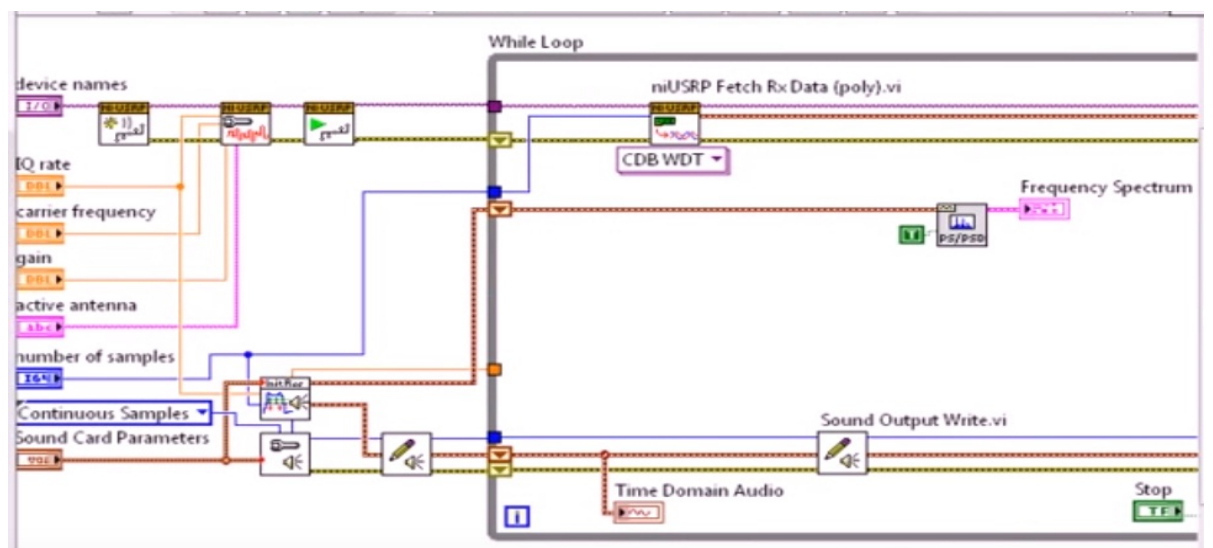

Fig. 8. VI Snippet of Amplitude and Frequency modulation using ni USRP Configuration

USRP is wide band $\mathrm{MHz}$ receiver and centered around 93.5 MHz. Each peak in the output is local FM station. By zooming in with graph palette we can see different FM stations occupying a bandwidth of 200KHZ. The IQ Sampling rate can be changed according to user. The input is taken from sound card. The demodulation is done by using phase wrapping icon. The final operation resample's the original signal with $50 \mathrm{~K}$ samples. The frequency spectrum can also be plotted by the user specification. By tuning the frequency at the receiver the user can able to listen sound file directly.

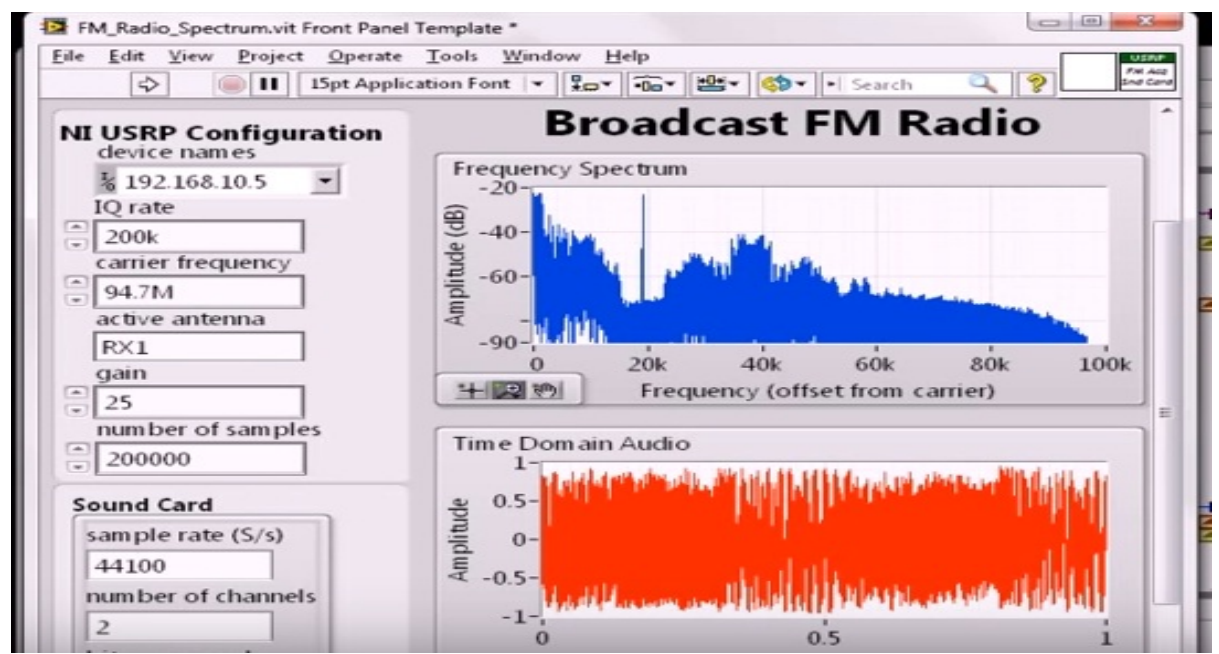

Fig. 9. USRP RIO Simulation results

Fig 9 gives the idea of receiving antenna with operating frequency. Here the 2 channels are used and the sampling rate is around $45 \mathrm{~K}$. the IQ rate is specified by the user. 


\section{Conclusion}

In this short paper the implementation of AM and FM modulation techniques are successfully carried out using NI USRP RIO 2920 and LabVIEW. By observing the results obtained we can conclude that demodulation of the modulated signal can be achieved successfully using Software defined radio receiver which is tuned to operating frequency. The amplitude modulation receiver is designed with the carrier frequency of $1560 \mathrm{KHz}$ and same design is implemented for FM receiver with carrier frequency of $94 \mathrm{MHZ}$.

\section{References}

[1] Lucky, R.W. Sal, J. Weldon (1968), Principles of Data Communication. McGraw-Hill, New York.

[2] Schwartz, M. (1990), Information Transmission, Modulation and Noise, McGraw-Hill, New York.

[3] K.Sharma, A.Mishra, Rajiv Saxena (2010). Analog \& Digital Modulation Techniques: An overview. International Journal of Computing Science and Communication Technologies. July Vol.3, No.1.

[4] C. Uluisik, L. Sevgi. (2012). A LabVIEW-Based Analog Modulation Tool for Virtual and Real Experimentation, IEEE Antenna and Propagation Magazine, December Vol 54, No 6.

[5] Korrapati, Raghu B, Swain, Nikunja K. (2000). Study of Modulation Using Virtual Instruments. Allied Academies International Conference. Academy of Information and Management Sciences.Proceedings;Arden, vol 4.1, pp-78-83.

[6] Ji Shujiao, Zhu Ming, Lei Yanmin (2013). The Simulation Design of Communication System Based on Lab VIEW. $2^{\text {nd }}$ International Conference on Measurement, Information and Control.

[7] Telagam, N., Kandasamy, N., \& Nanjundan, M. (2017). Smart Sensor Network Based High Quality Air Pollution Monitoring System Using Labview. International Journal of Online Engineering (iJOE), 13(08), 79-87. https://doi.org/10.3991/ijoe.v13i08.7161

\section{Authors}

Nehru Kandasamy (Corresponding Author) received his Bachelor Degree in Erode Sengunthar Engineering College, Anna University in 2005. And he obtained his Master Degree in R.M.K Engineering, Anna University in 2007.And he also obtained outstanding master student at that year. He is obtained his $\mathrm{Ph} . \mathrm{D}$ in 2014 at Faculty of Information and Communication Engineering, Anna University, India. His main research interest is in the area of Low Power VLSI, Testing of VLSI Circuits, FPGA Design, CAD for VLSI, Signal processing. He published 25 papers in Scopus Indexed journals. (nnehruk@gmail.com).

Nagarjuna Telagam is with the Electronics and Communication Engineering Department, Institute of Aeronautical Engineering, Hyderabad, India. Currently he is Ph.D Research Scholar in Sathyabama University, Chennai and is interested in the following topics: Wireless Communications, MIMO, OFDM, GFDM. Currently, He 
is working as Assistant Professor. He received his Bachelor degree from JNTU University/ Narayana Engineering College in 2011. He received his Master degree from Anna University/ Loyola Institute of Technology in 2013. He is Anna University rank holder (20) for M.E degree in 2013.He has nearly 4 years of experience in teaching and research. He published 15 papers in Scopus Indexed Journals. He is Certified LabVIEW Associate Developer. (nagarjuna473@gmail.com).

Prof. V R Seshagiri Rao works as head of department in Electronics and Communication Engineering, Institute of Aeronautical Engineering, Hyderabad, India. Currently he is pursing Ph.D in JNTU Hyderabad. His area of specialization is Fault Tolerance Systems. He has 31 years of experience in teaching and research. (vadrevu.vr@gmail.com)

Dr. T.S.Arulananth is with department of Electronics and communication Engineering, MLR institute of technology, Hyderabad, India. His areas of specialization is image processing, Embedded systems and Communication systems. He published 30 papers in Scopus indexed journals. He also published two books for undergraduate students in Anna university Chennai. (arulanandh.ts@gmail.com).

Article submitted 08 August 2017. Published as resubmitted by the authors 15 September 2017. 